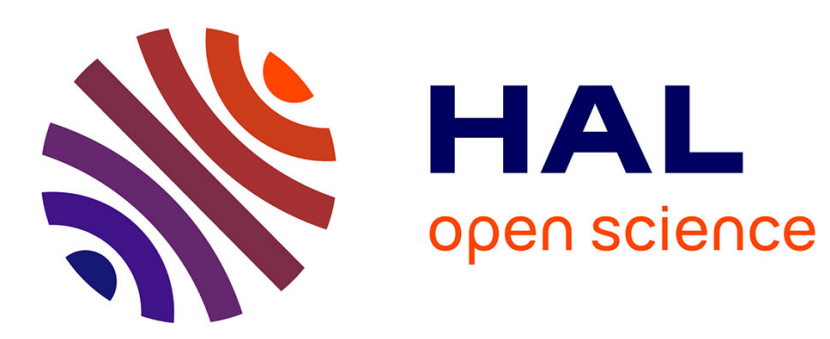

\title{
Parametric identification of nonlinear hysteretic systems
}

\author{
Youssef Rochdi, Fouad Giri, F. Ikhouane, Fatima Zara Chaoui, J. Rodellar
}

\section{To cite this version:}

Youssef Rochdi, Fouad Giri, F. Ikhouane, Fatima Zara Chaoui, J. Rodellar. Parametric identification of nonlinear hysteretic systems. Nonlinear Dynamics, 2009, 58 (1), pp.393-404. 10.1007/s11071-0099487-y . hal-01058885

\section{HAL Id: hal-01058885 \\ https://hal.science/hal-01058885}

Submitted on 28 Aug 2014

HAL is a multi-disciplinary open access archive for the deposit and dissemination of scientific research documents, whether they are published or not. The documents may come from teaching and research institutions in France or abroad, or from public or private research centers.
L'archive ouverte pluridisciplinaire HAL, est destinée au dépôt et à la diffusion de documents scientifiques de niveau recherche, publiés ou non, émanant des établissements d'enseignement et de recherche français ou étrangers, des laboratoires publics ou privés. 


\title{
Parametric identification of nonlinear hysteretic systems
}

\author{
Y. Rochdi • F. Giri · F. Ikhouane · F.Z. Chaoui • \\ J. Rodellar
}

\begin{abstract}
The hysteretic behavior is an essential feature of many physical systems (e.g. mechanical structures, buildings dampers). Such a feature is conveniently accounted for in hysteretic systems' modeling through the well-known Bouc-Wen equations. But these involve several unknown parameters and internal signals that are not all accessible to measurements. Furthermore, not all parameters come in linearly. All these difficulties make the identification of hysteretic systems a challenging problem. To cope with these issues, previous works have simplified the problem by supposing that the system displacements are large, the restoring force (and other internal signals) are accessible to measurements, the displacement is the actual control signal, the unknown parameter entering nonlinearly is known or is an integer, etc. In fact, these restrictive assumptions amount to supposing, among others, that the Bouc-Wen equations describe an isolated physical element in which 'hysteretis' is the only dynamic feature. The point is that the control input should be an external driving force and not the displacement. In this paper, the hysteretic equations are let to be what they really are in most practical situations: just a part of the system dynamics. Such a more
\end{abstract}

Y. Rochdi · F. Giri (凶) · F.Z. Chaoui

GREYC, University of Basse-Normandie, Caen, France

e-mail: fouad.giri@unicaen.fr

F. Ikhouane $\cdot$ J. Rodellar

Universitat Politècnica de Catalunya, Barcelona, Spain realistic problem statement has a cost, which is in additional unknown parameters. A multi-stage parametric identification scheme is designed in this paper and shown to recover consistently the unknown system parameters. The proposed solution is suitable for systems not tolerating large displacements (e.g. buildings) as well as for situations where force, velocity and acceleration sensors are not available.

Keywords Nonlinear system identification . Hysteretic systems · Bouc-Wen model

\section{Introduction}

Hysteresis is a memory feature that characterizes a wide variety of nonlinear systems. In these systems, the output value at a given time instant depends not only on the instantaneous input but also on its past history. To describe the behavior of hysteretic processes, several mathematical models have been proposed, e.g. Preisach and Bouc-Wen models [4]. In this paper the focus is made on the Bouc-Wen model because its full parametric nature makes it suitable for parametric identification. In practical applications, such model describes softening hysteretic behavior [7]. It may also represent a quasi-linear hysteretic behavior that is compatible with certain physical phenomena, e.g. thermodynamic laws [9]. Nevertheless, the BoucWen model has received a great deal of interest due 
to its numerical tractability and its ability to match the behavior of a wide class of systems in mechanical and structural engineering. In particular, it has been used to model piezoelectric actuators, base-isolation devices and magnetorheological dampers $[5,6,8]$. In this model, the restoring force $\Phi_{\mathrm{BW}}(t)$ is related to the displacement $x(t)$ as follows:

$$
\begin{aligned}
\Phi_{\mathrm{BW}}(t) & =\alpha k x(t)+(1-\alpha) D k z(t), \\
\dot{z}(t)= & D^{-1}\left(A \dot{x}(t)-\beta|\dot{x}(t)||z(t)|^{n-1} z(t)\right. \\
& \left.-\gamma \dot{x}(t)|z(t)|^{n}\right)
\end{aligned}
$$

where the parameters $0<\alpha<1, k>0, D>0$, $A>0,-\beta<\gamma \leq \beta, n \geq 1$ determine the shape and size of the hysteresis loop. Identification of the model (1)-(2) amounts to determining the parameters $\alpha, \beta, \gamma, A, D, k, n$. This is not a trivial task as the state $z(t)$ is not accessible to measurements. Furthermore, the parameter $n$ comes nonlinearly in the model and is not necessarily an integer. Many identification methods have been proposed to get estimates of the BoucWen model parameters. A number of early methods have been reported in [7] and more recent ones have been presented in $[1,3,8]$. However, the proposed methods are generally based on simplifying assumptions on the system and on the signals. For instance, in [1] and [8] it was supposed that some system parameters (e.g. system mass, friction coefficient) are known and the second derivative of $x(t)$ is measurable. In [1], it was assumed that $n$ is an integer and bounded by a known integer. In [3] it is supposed that the restoring force $\Phi_{\mathrm{BW}}(t)$ is accessible to measurements and the displacement $x(t)$ is the actual control input of the system.

In this paper, the hysteretic element (1)-(2) is assumed to be a part of a more complete mechanical structure of single-degree-of-freedom (SDOF). Accordingly, the displacement $x(t)$ undergoes a Newton motion equation driven by an external force. Furthermore, the displacement measurement is affected by an additive noise. The considered SDOF structure is controlled by the driving force (input signal) and observed by the (noisy) measured displacement (output signal). The more realistic system thus defined is characterized by (1)-(2), together with the Newton equation and output equations. The Newton equation introduces additional unknown parameters (inertia and friction coefficients). An identification scheme is designed to get estimates of all unknown parameters without resorting to those unrealistic assumptions, such as supposing accessible to measurement the restoring force and the displacement derivatives or supposing the parameter $n$ to be an integer. The proposed method operates in three main stages. In each stage a part of the unknown parameters and unmeasured signals is estimated and the estimates obtained in one stage are based upon in the next stage to estimate other signals and parameters. The proposed identification method relies on simple experiments that only necessitate sine wave exciting signals. Finally, note that consistency properties are formally established for all involved estimators (while earlier works resorted generally to simulations to illustrate consistency $[1,7,8]$ ).

The paper is organized as follows: the identification problem at hand is formulated in Sect. 2; the proposed identification method is designed and analyzed in Sect. 3. Simulations illustrating the given identification scheme efficiency are presented in Sect. 4. A conclusion and a reference list end the paper.

\section{Identification problem statement}

\subsection{Class of identified systems}

We are interested in (mechanical/structural) systems that involve the hysteresis feature. This is for instance the case of base isolators installed to supply passive or active (through actuators) protection of huge buildings against earthquakes. The motion of the base is described applying Newton's law:

$m \ddot{x}(t)+f \dot{x}(t)+\Phi_{\mathrm{BW}}(t)=u(t)$,

$x_{d}(t)=x(t)+\xi(t)$

where $u(t)$ represents the excitation force; $x(t)$ denotes the real displacement and $x_{d}(t)$ is its measured value; $\xi(t)$ is a zero-mean ergodic stochastic process accounting for external disturbances. The constants $m$ and $f$ are respectively the inertia and the friction coefficient. $\Phi_{\mathrm{BW}}(t)$ denotes the nonlinear restoring force that is assumed to undergo the following normalized Bouc-Wen model:

$\Phi_{\mathrm{BW}}(t)=k_{x} x(t)+k_{w} \omega(t)$, 


$$
\begin{aligned}
\dot{\omega}(t)= & \rho\left(\dot{x}(t)-\sigma|\dot{x}(t)||\omega(t)|^{n-1} \omega(t)\right. \\
& \left.-(1-\sigma) \dot{x}(t)|\omega(t)|^{n}\right)
\end{aligned}
$$

where

$$
\begin{aligned}
& k_{x}>0, \quad k_{w}>0, \quad \rho>0, \quad \sigma \geq \frac{1}{2} \\
& \text { and } \quad n \geq 1 .
\end{aligned}
$$

The normalized model (5)-(6) involves only five unknown parameters, while the initial model (1)-(2) involved seven parameters. Equation (5) shows that the restoring force $\Phi_{\mathrm{BW}}(t)$ is the superposition of an elastic component $k_{x} x(t)$ and a hysteretic component $k_{w} \omega(t)$. The signal $\omega(t)$, which is an internal state, is not supposed to be available. Consequently, the restoring force is in turn not available. In fact, $u(t)$ and $x_{d}(t)$ are the only measurable signals. In particular, $x(t)$ and its derivatives $\dot{x}(t)$ and $\ddot{x}(t)$ are not supposed to be measurable.

\section{Remark 2.1}

(1) The fact that none of $\Phi_{\mathrm{BW}}(t), x(t), \dot{x}(t)$ and $\ddot{x}(t)$ is supposed to be measurable does constitute a substantial progress with respect to the existing literature, e.g. $[1,3,8]$.

(2) A vibration system could be either force controlled or displacement controlled. In the last case, the internal signal $x(t)$ must be sufficiently exciting to ensure consistent parameter estimation. In [3], the internal signal $x(t)$ is supposed to be T-periodic of a specific shape. Such assumption amounts to neglect the mass $m$ in (3). The point is that, such an assumption does not hold in all situations. Therefore, the identification problem must be dealt with taking into account the full system described by the mechanical equations (3)-(4) and the hysteretic equations (5)-(6). Such system is driven by the external force $u(t)$ and has the displacement $x(t)$ as output.

(3) Another interesting feature of the present study, compared to most previously mentioned works, is that the identification scheme design is performed in presence of a measurement noise affecting the displacement. This makes the present identification design more realistic. In [8] a displacement measurement noise was only accounted for in the simulation stage.

\subsection{Identification objective}

Our purpose is to design an identification scheme that provides asymptotically accurate estimates of the unknown parameters $m, f, k_{x}, k_{w}, \rho, \sigma$ and $n$. From an identification viewpoint the difficulty does not lie only in the system memory feature, it also lies in the fact that most internal signals $\left(\omega(t), \Phi_{\mathrm{BW}}(t), x(t)\right.$, $\dot{x}(t), \ddot{x}(t))$ are not accessible to measurement (i.e. system identification should only be based on the control input $u(t)$ and the output measure $\left.x_{d}(t)\right)$. Another difficulty lies in the fact that the unknown parameter $n$ is not necessarily an integer and comes in nonlinearly in (6).

\section{Identification scheme design}

\subsection{Hysteretic model re-parameterization}

The proposed identification scheme is based on a new re-parameterization of the system. To this end, we rewrite (5) and (7) as follows:

$$
\begin{aligned}
& \Phi_{\mathrm{BW}}(t)=k_{x} x(t)+v(t), \\
& \dot{v}(t)=a \dot{x}(t)-b|\dot{x}(t)||v(t)|^{n-1} v(t)+c \dot{x}(t)|v(t)|^{n}
\end{aligned}
$$

where:

$v(t)=k_{w} \omega(t)$,

$a=\rho k_{w} ; \quad b=\frac{\rho \sigma}{k_{w}^{n-1}} ; \quad c=\frac{\rho(\sigma-1)}{k_{w}^{n-1}}$.

In this re-parameterized model, the unknown parameters are $n, m, f, k_{x}, a, b$, and $c$. The initial parameters $k_{w}, \rho, \gamma$ can be recovered from the new parameters $a, b, c$ and $n$ using the following equations:

$k_{w}=\left(\frac{a}{b-c}\right)^{1 / n} ; \quad \rho=\frac{a}{k_{w}} ; \quad \sigma=\frac{b}{a}\left(k_{w}\right)^{n}$.

\subsection{Estimation of the true displacement $x(t)$}

All studies on Bouc-Wen model lie on the experimentally-based premise that a steady-state hysteretic limit cycle is obtained whenever the considered hysteretic 
element is excited by a periodic input signal see e.g. $[1,8]$. The only existing formal proof of this fact is due to Ikhouane and Rodellar [2] and concerns a specific class of periodic input signals. The involved signals are mainly featured by their monotonic variation in $[k T k T+\tau]$ and opposite-sense monotonic variation in $[k T+\tau(k+1) T]$, for some constant $\tau(0<\tau<T)$, where $T$ is the signal period and $k=0,1,2, \ldots$ It is clear that the cosine signals, which are widely used in the present paper for their simplicity, do share such feature. Furthermore, it is proved that the hysteretic limit cycle is fully determined by the input signal i.e. is independent of the initial conditions. This implies that, in presence of a periodic input signal, the undisturbed output is in turn periodic in steady state (with the same period as the input) and is independent of the initial conditions (it only depends on the input).

The proposed identification scheme necessitates five experiments: two in the first stage of the identification scheme (during the SSO procedure), two in the second stage, and one in the third stage. All of these involve simple periodic input signals, namely cosine waves. Consequently, in all experiments, the undisturbed output $x(t)$ turns out to be, in steady state, a periodic signal with the same period as the input $u(t)$. This property is now used to perform a consistent estimation of $x(t)$, using the measured signal $x_{d}(t)$. In effect, letting $T$ denote the period of the input signal, the displacement $x(t)$ can be estimated as follows:

$$
\begin{gathered}
\bar{x}(t, N)=\frac{1}{N} \sum_{i=1}^{N} x_{d}(t+i T) \quad \text { for } 0 \leq t<T, \\
\bar{x}(t, N)=\bar{x}(t-k T, N) \\
\text { for } k T \leq t<(k+1) T, k=1,2,3, \ldots
\end{gathered}
$$

Proposition 3.1 The estimator $\bar{x}(t, N)$ (for a given, but arbitrary $t$ ) is consistent provided that $N$ is sufficiently large. More precisely, one has w.p. 1: $\bar{x}(t, N) \underset{N \rightarrow \infty}{\rightarrow} x(t)$ (for all $t)$.

Proof Substituting (3) into (12) one has, successively:

$$
\begin{aligned}
\bar{x}(t, N) & =\frac{1}{N} \sum_{i=1}^{N} x(t+i T)+\frac{1}{N} \sum_{i=1}^{N} \xi(t+i T) \\
& =x(t)+\frac{1}{N} \sum_{i=1}^{N} \xi(t+i T)
\end{aligned}
$$

using the periodicity of $x(t)$. But, the ergodicity of $\xi(t)$ implies that

$$
\frac{1}{N} \sum_{i=1}^{N} \xi(t+i T) \rightarrow E(\xi(t))=0
$$

(w.p. 1) as $N \rightarrow \infty$.

The above equalities establish the consistency of (12)

\subsection{Outline of the proposed identification scheme}

A general view of the proposed identification scheme is sketched here. Roughly speaking, the proposed identification scheme includes three main steps.

First, the parameters $m$ and $f$ will be estimated and the estimates will be denoted $\hat{m}$ and $\hat{f}$. Also, the derivatives $\dot{x}(t)$ and $\ddot{x}(t)$ will be estimated, from $\bar{x}(t, N)$, and the estimates will be denoted $\dot{\bar{x}}(t, N)$ and $\ddot{\ddot{x}}(t, N)$. Then, an obvious estimator of $\Phi_{\mathrm{BW}}(t)$ will simply be:

$\hat{\Phi}_{\mathrm{BW}}(t, N)=u(t)-\hat{m} \ddot{\bar{x}}(t, N)-\hat{f} \dot{\bar{x}}(t, N)$.

In the second step, the parameter $k_{x}$ is estimated and the estimate $\hat{k}_{x}$ will be used in the following estimator of the internal variable $v(t)$ :

$\hat{v}(t, N)=\hat{\Phi}_{\mathrm{BW}}(t, N)-\hat{k}_{x} \bar{x}(t, N)$.

In the third step, the rest of the parameters (i.e. $a, b, c, n)$ are estimated based on (9), using $\hat{v}(t, N)$. The estimates thus obtained will in turn be used to get estimates for the parameters $k_{w}, \rho, \gamma$ making use of (11).

\subsection{Estimation of $m, f$ and $\Phi_{\mathrm{BW}}(t)$}

As already mentioned, we only use, in this paper, cosine input signals i.e.

$u(t)=U_{m} \cos (\omega t)$.

It also has been mentioned earlier that the resulting (steady-state) displacement $x(t)$ is periodic with pe$\operatorname{riod} T=2 \pi / \omega$. Then, invoking the Fourier series theory, the displacement can be decomposed as follows:

$x(t)=X_{1} \cos (\omega t-\varphi)+x_{\mathrm{har}}(t)$ 
with

$x_{\text {har }}(t)=\sum_{h=2}^{\infty} X_{h} \cos \left(h \omega t-\varphi_{h}\right)$.

That is, $x_{\text {har }}(t)$ includes higher order harmonics resulting from the system nonlinearity (accounted for through (5)-(6)). Furthermore, it can be shown (see e.g. [2]) that for small displacements the relationship between $\Phi_{\mathrm{BW}}(t)$ and $x(t)$ becomes linear in steady state, i.e.:

$\Phi_{\mathrm{BW}}(t)=\left(k_{x}+a\right) x(t)$.

This means that for a periodic displacement with small magnitude the hysteretic feature has no substantial effect on the displacement. In view of (17), (3) becomes:

$m \ddot{x}(t)+f \dot{x}(t)+K x(t) \cong u(t)$

with

$K=k_{x}+a$.

The smaller is $\max _{t}|x(t)|$, the more accurate is the linear equation (18a). This in turn implies that $x_{\text {har }}(t) \rightarrow$ 0 as $\max _{t}|x(t)| \rightarrow 0$.

The second-order linear equation (18a) is particularly suitable to get estimates of the unknown parameters $m, f, K$. But, it is important to recall that $x(t)$, which represents the output of the system is not directly measurable. So, the question is: How to check that the validity conditions of such equation are actually fulfilled?

To answer this question, we make use of the fact that $\bar{x}(t, N)$ is a consistent estimate of the (unavailable) signal $x(t)$ and the fact that $x_{\text {har }}(t) \rightarrow 0$ as $\max _{t}(x(t)) \rightarrow 0$. The idea is to drive the system into a 'small-signal' operation regime, by tuning $U_{m}$ (and possibly $\omega$ ) and observing the size of $x_{\text {har }}(t)$. This is precisely formulated in the following search procedure:

\section{Small signal operation ( $\mathrm{SSO}$ )}

(1) Develop the T-periodic signal $\bar{x}(t, N)$ in Fourier series.

(2) Compute the distortion ratio: $D_{R}=\frac{\sum_{h}\left(\bar{X}_{h}\right)^{2}}{\bar{X}_{1}^{2}}$.

(3) If $D_{R}>\varepsilon$, then tune $U_{m}$ (or $\left.\omega\right)$ so that $\max _{t}|x(t)|$ decreases and go to Step 1. Else, note the values of $U_{m}, \omega$ and $\bar{X}_{1}$ and end the procedure.
In Step 3, the parameter $\varepsilon$ is a real threshold whose choice is left to the designer. The outcome of the SSO procedure is a quadruplet $\left(U_{m}, \omega, \varphi(\varepsilon), \bar{X}_{1}(\varepsilon)\right)$ corresponding to conditions where (17)-(18a) hold with an error that depend on $\varepsilon$. The smaller $\varepsilon$ is, the more accurate (17)-(18a) are. However, a too small value of $\varepsilon$ will necessitate a too long time for the SSO search procedure to end. Therefore, $\varepsilon$ should be chosen bearing in mind the above two requirements. Our simulations has shown that the choice $\varepsilon=0.05$ is a satisfactory compromise. The above procedure can be run on several times leading to different quadruplets. Let $\left(U_{m 1}, \omega_{1}, \varphi_{1}(\varepsilon), \bar{X}_{11}(\varepsilon)\right)$ and $\left(U_{m 2}, \omega_{2}, \varphi_{2}(\varepsilon), \bar{X}_{12}(\varepsilon)\right)$ denote two of such quadruplets.

On the other hand, the following expressions readily follow from (18a):

$\left|\frac{\bar{X}_{1}}{U_{m}}\right|=\frac{1}{\sqrt{(f \omega)^{2}+\left(K-m \omega^{2}\right)^{2}}}$,

$\tan (\varphi)=\frac{f \omega}{K-m \omega^{2}}$.

In effect, these characterize the harmonic behavior of any linear second-order system. Substituting, successively $\left(U_{m 1}, \omega_{1}, \varphi_{1}(\varepsilon), \bar{X}_{11}(\varepsilon)\right)$ and $\left(U_{m 2}, \omega_{2}, \varphi_{2}(\varepsilon)\right.$, $\left.\bar{X}_{12}(\varepsilon)\right)$ into the above expressions, one gets four equations involving the unknown parameters $m, f$, and $K$. Then estimates $\hat{m}(\varepsilon), \hat{f}(\varepsilon), \hat{K}(\varepsilon)$ can be obtained solving the obtained equations. The quality of the estimates depends on the value of $\varepsilon$. The smaller $\varepsilon$ is, the more accurate the estimates. The result thus established is simply formulated as follows:

$\hat{m}(\varepsilon)=m+O(\varepsilon) ; \quad \hat{f}(\varepsilon)=f+e(\varepsilon)$,

$\hat{K}(\varepsilon)=K+e(\varepsilon)$

for some function $e(\varepsilon)$ that vanishes when $\varepsilon \rightarrow 0$.

\section{Remark 3.1}

(1) In [3], no method has been given to check whether condition (18a) holds or not. In the light of these observations, the procedure SSO presented above turns out to be a significant progress.

(2) On the other hand, since the signal $x(t)$ and its derivatives $\dot{x}(t)$ and $\ddot{x}(t)$ are presently inaccessible to measurement, the estimation of the pa- 
rameters $m, f, K$ using the least-squares estimator based on the parameterization equation (18a) would not be possible.

(3) Now, it is clear that our identification scheme also applies to the simpler case of displacement controlled system. Then, Sects. 3.2 (estimation of $x$ ) and 3.4 (estimation of $m$ and $f$ ) are skipped.

\subsection{Estimation of $k_{x}, a$ and $v(t)$}

In this section, the amplitude of the output signal $x(t)$ is no longer supposed to be small. In such a case, the curve $(u(t), \bar{x}(t))$ leads to a visible hysteretic limit cycle. Making adequate use of such limit cycles, it will be possible to get estimates of $k_{x}$ and $a$. These estimates will in turn be used to build up an estimator of the internal state $v(t)$.

Estimation of $k_{x}$ and $a$. To this end, we perform two experiments that consist in exciting the system, successively, with the two following signals:

$u_{1}(t)=U_{m} \cos (2 \pi t / T)$,

$u_{2}(t)=U_{m} \cos (2 \pi t / T)+U$

where $U_{m}$ and $U$ are any real constants (with $U \neq 0$ and $U_{m} \neq 0$ ). Let $x_{1}(t)$ and $x_{2}(t)$ denote the resulting (undisturbed) displacements. These are $T$-periodic because the input signals $u_{1}(t)$ and $u_{2}(t)$ are so. As mentioned earlier in this section, the steady-state parts of $x_{1}(t)$ and $x_{2}(t)$ are unique and independent of the initial conditions; they are fully determined by $u_{1}(t)$ and $u_{2}(t)$ respectively. Now, one has the following result.

Proposition 3.2 Consider the system described by (3)-(4) and (8)-(9). Let $\left(x_{1}(t), v_{1}(t)\right)$ and $\left(x_{2}(t), v_{2}(t)\right)$ be the solutions obtained when applying $u_{1}(t)$ and $u_{2}(t)$, respectively. Then one has, for all sufficiently large $t$ :

$x_{1}(t)=x_{2}(t)+\frac{U}{k_{x}} \quad$ and $\quad v_{1}(t)=v_{2}(t)$.

Proof Let $\Delta$ be any real constant. Then, any couple of signals $(x(t), v(t))$ such that:

$x(t)=x_{1}(t)+\Delta, \quad v(t)=v_{1}(t)$

(for all sufficiently large $t$ ) undergoes the differential equation (9). Indeed, one has successively (for all sufficiently large $t$ ):

$$
\begin{aligned}
a \dot{x}(t) & -b|\dot{x}(t)||v(t)|^{n-1} v(t)+c \dot{x}(t)|v(t)|^{n} \\
= & a\left(\dot{x}_{1}(t)+\dot{\Delta}\right)-b\left|\dot{x}_{1}(t)+\dot{\Delta}\right|\left|v_{1}(t)\right|^{n-1} v_{1}(t) \\
& +c\left(\dot{x}_{1}(t)+\dot{\Delta}\right)\left|v_{1}(t)\right|^{n} \\
= & a\left(\dot{x}_{1}(t)\right)-b\left|\dot{x}_{1}(t)\right|\left|v_{1}(t)\right|^{n-1} v_{1}(t) \\
& +c\left(\dot{x}_{1}(t)\right)\left|v_{1}(t)\right|^{n} \\
& \quad(\text { using the fact that } \Delta \text { is constant }) \\
= & \dot{v}_{1}(t) \quad\left(\text { since }\left(x_{1}(t), v_{1}(t)\right) \text { satisfies }(9)\right) \\
= & \dot{v}(t) .
\end{aligned}
$$

Furthermore, substituting (22) into (8) one gets (for all sufficiently large $t$ ):

$$
\begin{aligned}
\Phi_{\mathrm{BW}}(t) & \stackrel{\text { def }}{=} k_{x} x(t)+v(t) \\
& =k_{x} x_{1}(t)+k_{x} \Delta+v_{1}(t) \\
& =\Phi_{\mathrm{BW}}^{1}(t)+k_{x} \Delta
\end{aligned}
$$

where

$\Phi_{\mathrm{BW}}^{1}(t) \stackrel{\text { def }}{=} k_{x} x_{1}(t)+v_{1}(t)$.

Using again the fact that $\left(x_{1}(t), v_{1}(t)\right)$ is the solution of (3), (8) and (9) when applying $u_{1}(t)$, it follows from (3) that:

$m \ddot{x}_{1}(t)+f \dot{x}_{1}(t)+\Phi_{\mathrm{WB}}^{1}(t)=u_{1}(t)$

which, together with (22) and (24)-(25), implies (for all sufficiently large $t$ ):

$m \ddot{x}(t)+f \dot{x}(t)+\Phi_{\mathrm{WB}}(t)=u_{1}(t)-k_{x} \Delta$.

At this point, $\Delta$ is arbitrary. Let us consider the choice $\Delta=U / k_{x}$. Then, using (21b), (26a) can be rewritten as (for all sufficiently large $t$ ):

$m \ddot{x}(t)+f \dot{x}(t)+\Phi_{\mathrm{WB}}(t)=u_{2}(t)$.

Equations (26b), (25), (23) show that the couple $(x(t), v(t))$ is a solution of the equation system $\{(3)$, (8), (9) $\}$ when this is excited by the input signal $u_{2}(t)$. But, we know that $\left(x_{2}(t), v_{2}(t)\right)$ is also a solution of that system (in response to $u_{2}(t)$ ). Furthermore, 
it was already noticed that the steady-state solution of that system is unique whatever the applied periodic input signal. Therefore, one has $x(t)=x_{2}(t)$ and $v(t)=v_{2}(t)$ (for all sufficiently large $t$ ). This, together with (22), implies that $x_{1}(t)=x_{2}(t)+\Delta$ and $v_{1}(t)=v_{2}(t)$ (for all sufficiently large $t$ ). Since $\Delta=U / k_{x}$, the proposition is established.

Proposition 3.2 suggests, for the parameter $k_{x}$, the following estimator:

$\hat{k}_{x}(N)=\frac{U}{\frac{1}{T} \int_{0}^{T}\left(\bar{x}_{2}(t, N)-\bar{x}_{1}(t, N)\right) d t}$

where $N$ is any sufficiently large integer. Also, (18b) suggests for the parameter $a$ the following estimator:

$\hat{a}(\varepsilon, N)=\hat{K}(\varepsilon)-\hat{k}_{x}(N)$.

Proposition 3.3 Let the system described by (3)-(4) and (8)-(9) be successively excited with the two input signals defined by (21a)-(21b). Let $x_{1}(t), x_{2}(t)$ denote the resulting displacements and $\bar{x}_{1}(t, N), \bar{x}_{2}(t, N)$ be their estimates obtained applying (12).

(1) The estimator (27) is consistent i.e. $\hat{k}_{x}(N) \rightarrow k_{x}$ (w.p. 1) as $N \rightarrow \infty$.

(2) The estimator (28) is consistent up to an error that depends on the threshold $\varepsilon$ introduced in the SSO procedure. More precisely, one has w.p. 1:

$$
\hat{a}(N, \varepsilon)-a \rightarrow e(\varepsilon) \quad \text { as } N \rightarrow \infty
$$

for some function $e(\varepsilon)$ that vanishes when $\varepsilon \rightarrow 0$.

Proof Proposition 3.1 assures that, for sufficiently large values of $t$, one has w.p. 1: $\bar{x}_{i}(t, N) \underset{N \rightarrow \infty}{\rightarrow} x_{i}(t)$ $(i=1,2)$. Then, the $T$-periodicity of $\bar{x}_{i}(t, N)(i=$ 1,2) implies that (w.p. 1):

$$
\begin{aligned}
& \frac{1}{T} \int_{0}^{T}\left(\bar{x}_{2}(t, N)-\bar{x}_{1}(t, N)\right) d t \\
& \quad \underset{N \rightarrow \infty}{\rightarrow} \frac{1}{T} \int_{0}^{T}\left(x_{2}(t+k T)-x_{1}(t+k T)\right) d t
\end{aligned}
$$

for any sufficiently large integer $k$. Using Proposition 3.2, it follows from (29) that

$\frac{1}{T} \int_{0}^{T}\left(\bar{x}_{2}(t, N)-\bar{x}_{1}(t, N)\right) d t \underset{N \rightarrow \infty}{\rightarrow} \frac{U}{k_{x}} \quad$ (w.p. 1)

which, together with (27), establishes part 1. Part 2 readily follows from part 1 , using (18b) and (20b).
Estimation of $v(t)$ If the derivatives $\dot{x}(t)$ and $\ddot{x}(t)$ were measurable, then a consistent estimator of $v(t)$ could readily be obtained from (3), (5) and (10a). Specifically, the obtained estimator would be:

$\hat{v}(t, N, \varepsilon)=u(t)-\hat{m}(\varepsilon) \ddot{x}(t)-\hat{f}(\varepsilon) \dot{x}(t)-\hat{k}_{x}(N) x(t)$.

However, accurate sensors of the displacement derivatives are generally not available in practice. Furthermore, it is generally not possible to design accurate estimators for arbitrary signal derivatives. Nevertheless, we will show that this is presently possible, thanks to the (steady-state) $T$-periodicity of the involved signals. Indeed, as $x(t)$ is periodic (in steady state) with period $T=2 \pi / \omega$, it can be developed in Fourier series:

$x(t)=x_{0}+\sum_{h=1}^{\infty} X_{h} \cos \left(h \omega t-\varphi_{h}\right)$.

The derivatives $\dot{x}(t)$ and $\ddot{x}(t)$ are in turn given the following Fourier expansions:

$\dot{x}(t)=-\sum_{h=1}^{\infty} h \omega X_{h} \sin \left(h \omega t-\varphi_{h}\right)$,
$\ddot{x}(t)=-\sum_{\mathrm{h}=1}^{\infty}(h \omega)^{2} X_{h} \cos \left(h \omega t-\varphi_{h}\right)$.

The above expressions show that if the Fourier coefficients $X_{h}$ of $x(t)$ were available, then it would be possible to obtain the derivatives $\dot{x}(t)$ and $\ddot{x}(t)$. The point is that the measurements $x_{d}(t)$ of $x(t)$ are noisy. Nevertheless, we do have a consistent estimator of $x(t)$, namely $\bar{x}(t, N)$. Then, instead of (31) we consider the Fourier series of $\bar{x}(t, N)$ :

$\bar{x}(t, N)=\bar{x}_{0}(N)+\sum_{h=1}^{\infty} \bar{X}_{h}(N) \cos \left(h \omega t-\varphi_{h}(N)\right)$.

Then, deriving both sides of (33) with respect to $t$ and truncating the obtained developments, one gets the following estimators for $\dot{x}(t)$ and $\ddot{x}(t)$ :

$$
\dot{\bar{x}}(t, N, M)=-\sum_{h=1}^{M} h \omega \bar{X}_{h}(N) \sin \left(h \omega t-\varphi_{h}(N)\right),
$$


$\ddot{\bar{x}}(t, N, M)=-\sum_{h=1}^{M}(h \omega)^{2} \bar{X}_{h}(N) \cos \left(h \omega t-\varphi_{h}(N)\right)$

where $M$ is any positive integer. Given the above estimates of $\dot{x}(t)$ and $\ddot{x}(t)$, (30) suggests the following estimator of $v(t)$ :

$$
\begin{aligned}
\hat{v}(t, N, M, \varepsilon)= & u(t)-\hat{m}(\varepsilon) \ddot{\ddot{x}}(t, N, M) \\
& -\hat{f}(\varepsilon) \dot{\bar{x}}(t, N, M)-\hat{k}_{x}(N) \bar{x}(t, N) .
\end{aligned}
$$

Proposition 3.4 The estimators (34a)-(34b) and (35) have the following properties:

(1) (34a)-(34b) are consistent, i.e.

(a) $\dot{\bar{x}}(t, N, M) \rightarrow \dot{x}(t)$ (w.p. 1) as $N, M \rightarrow \infty$.

(b) $\ddot{\bar{x}}(t, N, M) \rightarrow \ddot{x}(t)$ (w.p. 1) as $N, M \rightarrow \infty$.

(2) For sufficiently large t, (35) is consistent up to an error that depends on $\varepsilon$ (the threshold introduced in the procedure SSO). More precisely, we have:

$$
\begin{array}{r}
\hat{v}(t, N, M)-v(t) \rightarrow e(\varepsilon) \\
\quad \text { (w.p. 1) as } N, M \rightarrow \infty
\end{array}
$$

where $e(\varepsilon) \rightarrow 0$ if $\varepsilon \rightarrow 0$.

Proof It is readily seen from (33) and (34a)-(34b) that:

$\dot{\bar{x}}(t, N, M) \rightarrow \dot{\bar{x}}(t, N) \quad$ (w.p. 1) as $M \rightarrow \infty$,

$\ddot{\ddot{x}}(t, N, M) \rightarrow \ddot{\ddot{x}}(t, N) \quad$ (w.p. 1) as $M \rightarrow \infty$.

On the other hand, Proposition 3.1 ensures that:

$\bar{x}(t, N) \rightarrow x(t) \quad$ (w.p. 1) as $N \rightarrow \infty$.

The above equations imply parts $\mathrm{a}$ and $\mathrm{b}$.

Comparing (35) with (30), one gets part 2 using parts $\mathrm{a}-\mathrm{b}$ and (20a)-(20b).

Remark 3.2 The results of Proposition 3.3 suggest that the integers $N$ and $M$ should be sufficiently large. The larger these integers are, the better the quality of the estimates. As long as $M$ is concerned, (29) shows that a convenient choice is one that satisfies: $h^{2} X_{h} \ll X_{1}$ for all $h>M$.
3.6 Estimation of the remaining parameters

Though the signal $v(t)$ is accurately estimated, the estimation of the remaining parameters in (9) (namely, $b, c$ and $n$ ) is not that easy. In fact, one is faced with two major difficulties:

(i) Equation (9) involves the derivative of $v(t)$ and this is not accessible to measurements.

(ii) The parameter $n$ is not necessarily an integer and does not come in linearly.

One key idea to overcome these difficulties is to notice that (9) considerably simplifies in each one of the quadrants of the plane $(v(t), \dot{x}(t))$. More specifically, it follows from (9) that:

If $\dot{x}(t)>0$ and $v(t)>0$ then:

$\dot{v}(t)=\left[a-(b-c) v(t)^{n}\right] \dot{x}(t)$.

If $\dot{x}(t)>0$ and $v(t)<0$ then:

$\dot{v}(t)=\left[a+(b+c)|v(t)|^{n}\right) \dot{x}(t)$.

If $\dot{x}(t)<0$ and $v(t)<0$ then:

$\dot{v}(t)=\left[a+(-b+c)|v(t)|^{n}\right] \dot{x}(t)$.

If $\dot{x}(t)<0$ and $v(t)>0$ then:

$\dot{v}(t)=\left[a+(b+c) v(t)^{n}\right] \dot{x}(t)$.

These equations are to be considered together with (10a)-(10b). Now, let us consider the case where $\dot{x}(t)>0$ and $v(t)>0$. It follows from (36a) that:

$\frac{\dot{v}(t)}{\dot{x}(t)}-a=(c-b) v(t)^{n}$.

On the other hand, one gets from (10) that:

$c-b=-\frac{\rho}{k_{w}^{n-1}}=-\frac{a}{k_{w}^{n}}<0$.

Then, taking logarithms of both sides of (37a), yields:

$\log (-\vartheta(t)+a)=\log (b-c)+n \log (v(t))$,

$\vartheta(t) \stackrel{\text { def }}{=} \frac{\dot{v}(t)}{\dot{x}(t)}$.

Equation (38a) is quite interesting because: 
(i) The unknown parameters $n$ and $\log (b-c)$ come in linearly.

(ii) Accurate estimates are available for the involved signals, namely $\log (-\vartheta+a)$ and $\log (v)$.

Therefore, the unknown parameters can be recovered applying the least-squares estimator to the equation:

$$
\begin{aligned}
& \log (-\hat{\vartheta}(t, N, M, L, \varepsilon)+\hat{a}(N)) \\
& \quad=\log (b-c)+n \log (\hat{v}(t, N, M, \varepsilon))
\end{aligned}
$$

with

$\hat{\vartheta}(t, N, M, L, \varepsilon)=\frac{\hat{\dot{v}}(t, N, M, L, \varepsilon)}{\dot{\bar{x}}(t, N, M)}$

where $\hat{\dot{v}}(t, N, M, L, \varepsilon)$ denotes a consistent estimator (up to an error $e(\varepsilon)$ ) of the derivative $\dot{v}(t) . \hat{\dot{v}}(t, N, M$, $L, \varepsilon)$ is constructed making use of the fact that $v(t)$ is $T$-periodic (in steady state) and $\hat{v}(t, N, M, \varepsilon)$ is a consistent estimator (up to $O(\varepsilon)$ ). This construction is based on the Fourier series expansion of $\hat{v}(t, N, M, \varepsilon)$ up to the order $L$, just as this was done to get the estimators $\dot{\bar{x}}(t, N, M)$ and $\ddot{\bar{x}}(t, N, M)$ (see (34a)-(34b)) making use of the $T$-periodicity of $x(t)$ and the fact that $\bar{x}(t, N)$ is a consistent estimator. The least-squares estimator should be run on whenever $\dot{\bar{x}}(t, N, M)>0$ and $\hat{v}(t, N, M, \varepsilon)>0$. Doing so, one gets consistent estimates of $(b-c)$ and $n$.

Similarly, considering the case where $\dot{x}(t)>0$ and $v(t)<0$, it follows from (36b) and (10a) $-(10 \mathrm{~b})$ that:

$\frac{\dot{v}(t)}{\dot{x}(t)}-a=(c+b)|v(t)|^{n}$,

$c+b=\frac{\rho(2 \sigma-1)}{k_{w}^{n-1}} \geq 0 \quad$ (because $\sigma \geq 1 / 2$ ).

These imply:

$\log (\vartheta(t)-a)=\log (b+c)+n \log (|v(t)|)$.

There, too, the unknown parameters, $\log (b+c)$ and $n$, come in linearly and consistent estimators are available for the involved signals, i.e. $\vartheta(t)$ and $v(t)$. Then, the unknown parameters can be recovered applying the least-squares estimator to the equation:

$$
\begin{aligned}
& \log (\hat{\vartheta}(t, N, M, L, \varepsilon)-\hat{a}(N)) \\
& \quad=\log (b+c)+n \log (\hat{v}(t, N, M, \varepsilon)) .
\end{aligned}
$$

The present least-squares estimator is run on whenever $\dot{\bar{x}}(t, N, M)>0$ and $\hat{v}(t, N, M, \varepsilon)<0$. The consistent estimate thus obtained for $(b+c)$ is combined with that obtained previously for $(b-c)$ to get consistent estimates for $b$ and $c$.

\section{Evaluation of the identification scheme}

To illustrate the efficiency of the proposed identification scheme, we consider two different systems both described by (3)-(6). The parameters characterizing each system are given the following values:

System 1: $\rho=6 ; \quad n=2.7 ; \quad m=3 ; \quad f=7$;

$$
k_{x}=k_{w}=2 ; \quad \sigma=0.7 \text {; }
$$

System 2: $\quad \rho=3 ; \quad n=1.7 ; \quad m=1 ; \quad f=1.4$;

$$
k_{x}=k_{w}=2 ; \quad \sigma=1.7 \text {. }
$$

So the parameters to be identified, according to the system re-parameterization (8), are from (10), (18b) and (36c):

System 1: $a=12 ; \quad b=1.292 ; \quad c=-0.554$;

$$
K=14 \text {; }
$$

System 2: $\quad a=6 ; \quad b=3.139 ; \quad c=1.292$;

$$
K=8 \text {. }
$$

Both systems are disturbed by a sequence $\xi(t)$ of random numbers uniformly distributed over the interval [-0.04 0.04].

First stage: estimation of the parameters $m, f$ and $K$. Following Sect. 3.4, we successively apply (to each system) two sinusoidal input signals:

Signals applied to system 1:

$u_{1}(t)=0.4 \sin \left(\frac{\pi t}{20}\right) ; \quad u_{2}(t)=0.5 \sin \left(\frac{\pi t}{10}\right) ;$

Signals applied to system 2:

$u_{1}(t)=0.1 \sin \left(\frac{2 \pi t}{5}\right) ; \quad u_{2}(t)=0.2 \sin \left(\frac{4 \pi t}{5}\right)$.

Applying to each system the procedure described in Sect. 3.4, we obtain the estimates shown in Table 1, for two values of $N$. 
Table 1 Estimation of parameters $m, f, K$

\begin{tabular}{lllllll}
\hline & System 1 & & & \multicolumn{2}{c}{ System 2 } \\
\cline { 2 - 3 } & $m=3$ & $f=7$ & $K=14$ & & $f=1$ & $f .4$ \\
\hline$N=50$ & 3.534 & 7.201 & 14.075 & 1.045 & 1.475 & 8.266 \\
$N=200$ & 3.070 & 7.184 & 14.009 & 1.007 & 1.466 & 8.031 \\
\hline
\end{tabular}

Second stage: estimation of parameters $a$ and $k_{x}$. Following Sect. 3.4, the followings input signals are applied successively to each system:

Signals applied to system 1 :

$u_{1}(t)=2 \sin \left(\frac{\pi t}{10}\right) ; \quad u_{2}(t)=2 \sin \left(\frac{\pi t}{10}\right)+1 ;$

Signals applied to system 2 :

$u_{1}(t)=2 \sin \left(\frac{2 \pi t}{5}\right) ; \quad u_{2}(t)=2 \sin \left(\frac{2 \pi t}{5}\right)+1$.

Figures $1 \mathrm{a}-\mathrm{b}$ show the resulting filtered $\bar{x}_{1}(t, N)$ and $\bar{x}_{2}(t, N)$ obtained applying (12a)-(12b), with $N=200$, as well as the difference signal $\bar{x}_{2}(t, N)-$ $\bar{x}_{1}(t, N)$. Then, one gets using (28):

System 1: $\quad \hat{k}_{x}=\frac{U}{\left\langle\bar{x}_{2}-\bar{x}_{1}\right\rangle}=\frac{1}{0.5}=2 \quad$ and $\hat{a}=\hat{K}-\hat{k}_{x}=14.009-2=12.009$;

System 2: $\quad \hat{k}_{x}=\frac{U}{\left\langle\bar{x}_{2}-\bar{x}_{1}\right\rangle}=\frac{1}{0.5}=2$ and

$$
\hat{a}=\hat{K}-\hat{k}_{x}=8.031-2=6.031 \text {. }
$$

Third stage: estimation of the remaining parameters. Following Sect. 3.6, each system is excited by a sinusoidal input signal:

Signal applied to system 1: $\quad u_{3}(t)=2 \sin \left(\frac{\pi t}{10}\right) ;$

Signal applied to system 2: $\quad u_{3}(t)=2 \sin \left(\frac{2 \pi t}{5}\right)$.

Estimates of $\bar{x}(t, N), \quad \dot{\bar{x}}(t, N, M), \quad \ddot{\ddot{x}}(t, N, M)$, $\hat{v}(t, N, M, \varepsilon), \hat{\dot{v}}(t, N, M, \varepsilon, L)$ and $\hat{\vartheta}(t, N, M, L, \varepsilon)$ are obtained using (12), (30), (35) and (39b), and the following parameters:

System 1: $\quad N=800, \quad M=8, \quad L=8$

and $\varepsilon=0.05$;
System 2: $\quad N=200, \quad M=8, \quad L=8$

and $\varepsilon=0.05$.

Considering first system 1 , we plot the parameterized curve $(\log (\hat{v}(t, N, M, \varepsilon)), \log (-\hat{\vartheta}(t, N, M, L, \varepsilon)+$ $\hat{a}(N)))$ for those time instants $t$ such that $\dot{\bar{x}}(t, N, M)>$ 0 and $\hat{v}(t, N, M, \varepsilon)>0$. As expected the obtained plot (Fig. 2a) is a straight line (except for the borders due to computation errors). Then, performing a least-squares estimation (in the useful interval [ $-0.50 .45]$ ) we get the following estimates:

$\hat{n}=2.652$ and $\hat{b}-\hat{c}=1.877$.

Similarly, applying the least-squares estimator to (3.6), we get the estimates:

$\hat{n}=2.860$ and $\hat{b}+\hat{c}=0.662$.

Combining (43) and (44), one obtains the estimates:

$\hat{n}=(2.86+2.652) / 2=2.75, \quad \hat{b}=1.269$,

$\hat{c}=0.607$.

The remaining parameters are estimated using (11):

$\hat{k}_{w}=1.963 ; \quad \hat{\rho}=6.112 ; \quad \hat{\sigma}=0.675$.

Applying the same procedure for system 2, we first plot the curve $(\log (\hat{v}(t, N, M, \varepsilon)), \log (-\hat{\vartheta}(t, N, M$, $L, \varepsilon)+\hat{a}(N)))$, for all $t$ such that $\dot{\bar{x}}(t, N, M)>0$ and $\hat{v}(t, N, M, \varepsilon)>0$. The obtained plot yields (Fig. 2b):

$\hat{n}=1.598$ and $\hat{b}-\hat{c}=1.795$.

Then, the plot of $(\log (\hat{v}(t, N, M, \varepsilon)), \log (\hat{\vartheta}(t, N, M, L, \varepsilon)-$ $\hat{a}(N)))$ yields:

$\hat{n}=1.679$ and $\hat{b}+\hat{c}=4.278$. 


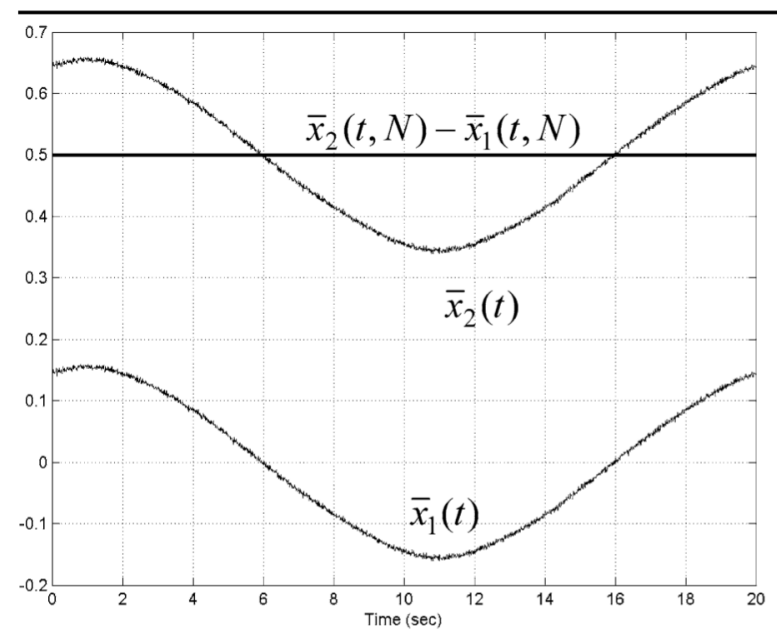

(a)

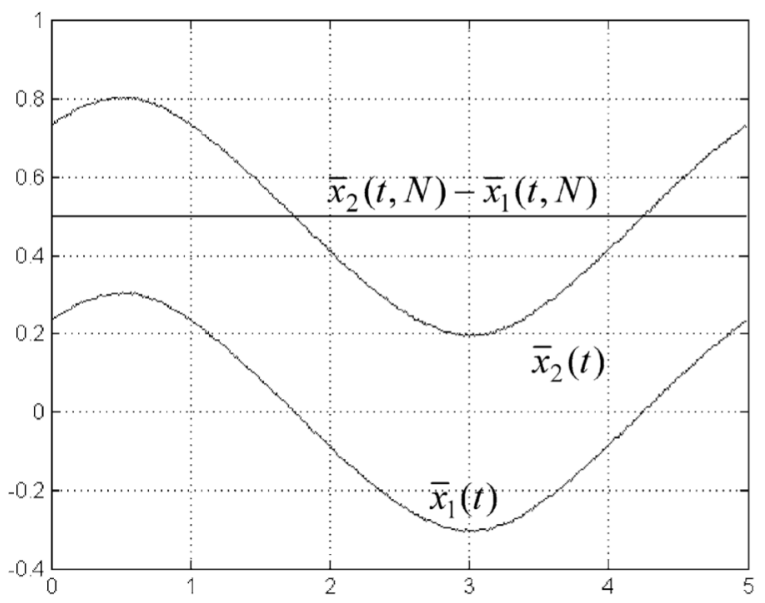

(b)

Fig. 1 (a) Estimation of the offset $\bar{x}_{2}-\bar{x}_{1}$ for system 1. (b) Estimation of the offset $\bar{x}_{2}-\bar{x}_{1}$ for system 2

Combining (45) and (46), one obtains:

$\hat{n}=(1.679+1.598) / 2=1.6385$,

$\hat{b}=3.036, \quad \hat{c}=1.241$.

Finally, using (11) one gets:

$\hat{k}_{w}=2.095 ; \quad \hat{\rho}=2.878 ; \quad \hat{\sigma}=1.742$.

It is readily checked that all obtained estimates are close to their true values, despite the presence of disturbances. This is further confirmed by Figs. 3a-b plotting the hysteretic limit cycles obtained by the true and the estimated models respectively.

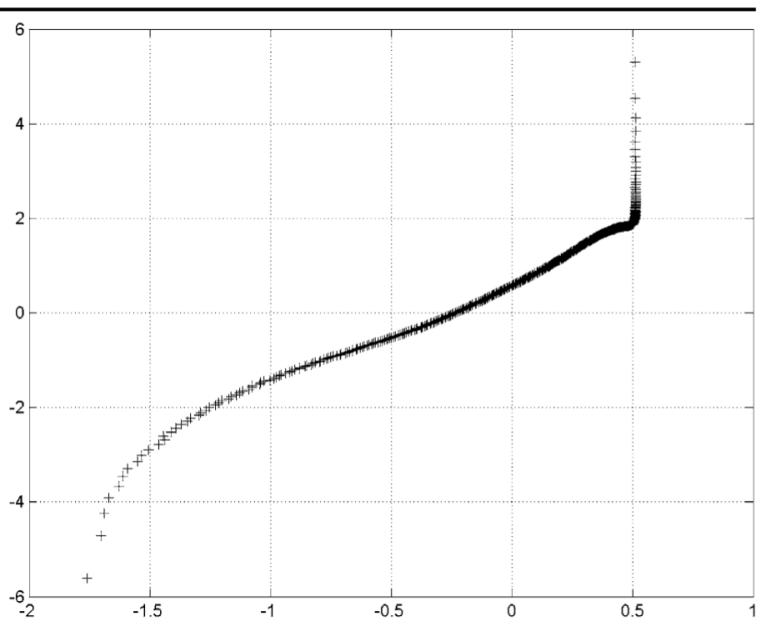

(a)

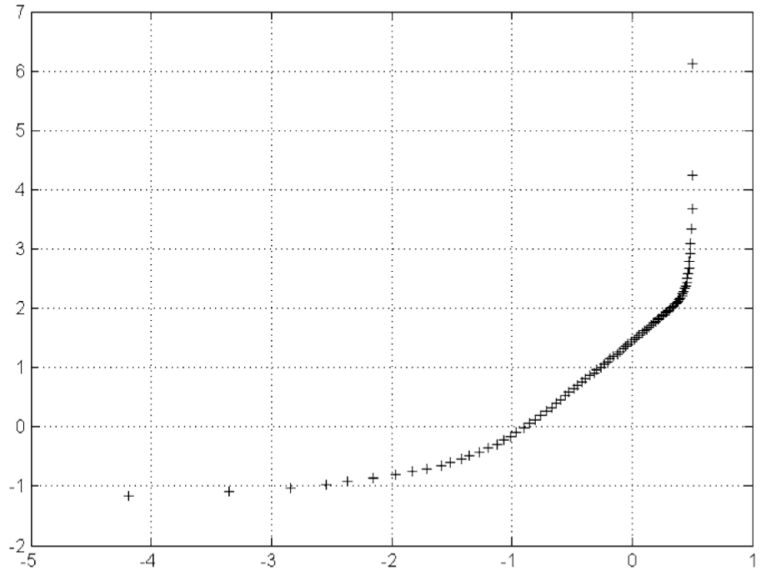

(b)

Fig. 2 (a) Plot of the curve $(\log (\hat{v}(t)), \log (-\hat{\vartheta}(t)+\hat{a}))$ for all $t$ such that $\dot{\bar{x}}(t)>0$ and $\hat{v}(t)>0$. (b) The plot $(\log (\hat{v}(t)), \log (\hat{\vartheta}(t)-\hat{a}))$ for all $t$ such that $\dot{\bar{x}}(t)<0$ and $\hat{v}(t)>0$

\section{Conclusion}

We have considered the problem of identifying systems whose dynamic behavior involves a hysteretic feature. Such a feature has been accounted for using the well-known Bouc-Wen equations. The system under study is described by (3)-(6) where the parameter $n$ comes in nonlinearly. Furthermore, the model involves internal signals that are not necessarily accessible to measurements (e.g. $\left.\Phi_{\mathrm{BW}}(t), v(t), \dot{x}(t), \ddot{x}(t)\right)$. Moreover, the displacement $x(t)$ is a state variable and so cannot stand for the system control input. The identification scheme we have designed, in Sect. 3, operates in three main steps. In each step, a part of 


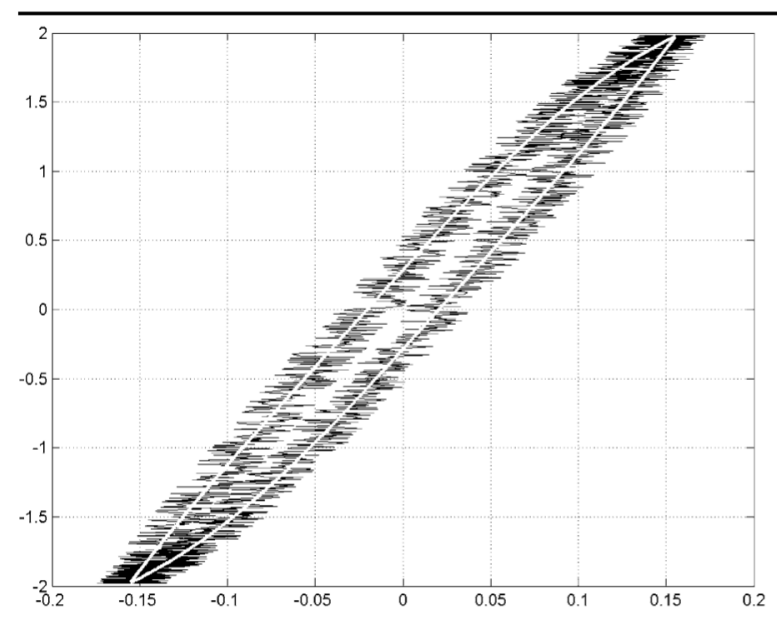

(a)

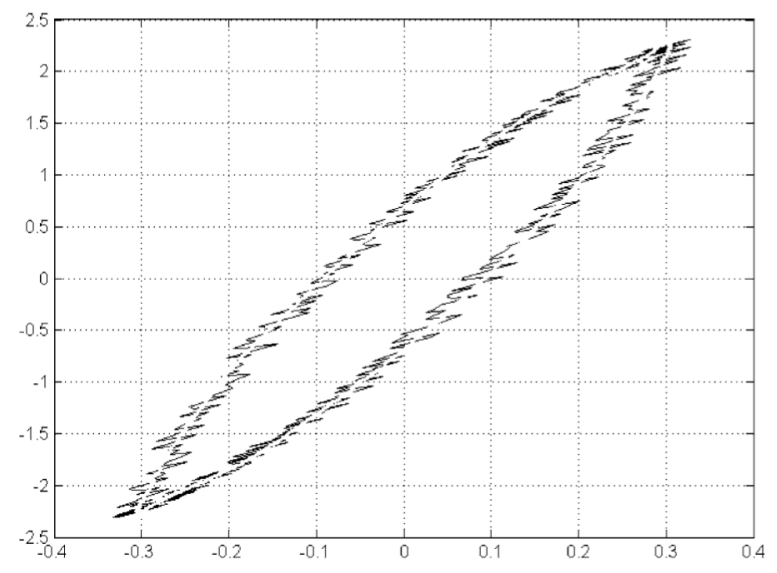

(b)

Fig. 3 (a) Limit cycles of system 1: true model cycle $\left(x_{d}(t), \Phi_{\mathrm{BW}}(t)\right)$ (black line) and estimated model cycle $\left(\bar{x}(t, N), \hat{\Phi}_{\mathrm{BW}}(t, N)\right)$ (white line). (b) Limit cycles of system 2 : true model cycle $\left(x_{d}(t), \Phi_{\mathrm{BW}}(t)\right)$ (black line) and estimated model cycle $\left(\bar{x}(t, N), \hat{\Phi}_{\mathrm{BW}}(t, N)\right)$ (white line)

the unknown parameters is estimated and the estimates are used in the next step to estimate other unknown parameters. The involved estimators are generally shown to be consistent. Compared to the few ex- isting works, our solution does not suppose the internal signals $\left(\Phi_{\mathrm{BW}}(t), v(t), \dot{x}(t), \ddot{x}(t)\right)$ to be available, or the displacements to be large or the parameter $n$ to be known and integer. Therefore, a model that is suitable for small as well as for large displacements is obtained without resorting to high price and inaccurate sensors. Moreover, the system actual control input is let be what it is in practice: the driving force $u(t)$. Finally, the proposed solution requires simple experiments as these only involve sine input signals. All these features represent a real progress in the topic of hysteretic system identification.

\section{References}

1. Smith, A.W., Masri, S.F., Chassiakos, A.G., Caughey, T.K.: On-line parametric identification of MDOF non-linear hysteretic systems. J. Eng. Mech. 125(2), 133-142 (1999)

2. Ikhouane, F., Rodellar, J.: On the hysteretic Bouc-Wen model. Part I: Forced limit cycle characterization. Nonlinear Dyn. 42, 63-78 (2005a)

3. Ikhouane, F., Rodellar, J.: On the hysteretic Bouc-Wen model. Part II: Robust parametric identification. Nonlinear Dyn. 42, 79-95 (2005b)

4. Macki, J.W., Nistri, P., Zecca, P.: Mathematical models for hysteresis. SIAM Rev. 35(1), 94-123 (1993)

5. Nagarajaiah, S., Xiaohong, S.: Response of base-isolated USC hospital building in Northridge earthquake. J. Struct. Eng. 126(10), 1177-1186 (2000)

6. Savaresia, S.M., Bittantia, S., Montigliob, M.: Identification of semi-physical and black-box non-linear models: The case of MR-dampers for vehicles control. Automatica 41, 113-127 (2005)

7. Wen, Y.K.: Method of random vibration of hysteretic systems. ASCE J. Eng. Mech. Div. 102(2), 249-263 (1976)

8. Ni, Y.Q., Ko, J.M., Wong, C.W.: Identification of non-linear hysteretic isolators from periodic vibration tests. J. Sound Vib. 217, 737-756 (1998)

9. Erlicher, S., Point, N.: Thermodynamic admissibility of Bouc-Wen type hysteresis models. C. R. Mecanique 332, 51-57 (2004)

10. Ma, F., Zhang, H., Bockstedte, A., Foliente, G.C., Paevere, P.: Parametric analysis of the differential model of hysteresis. J. Appl. Mech. 71, 342-349 (2004) 